\title{
Integrative taxonomy reveals that all the phenotype variations are not underpinned by genome in Conidae (Gastropoda, Mollusca)
}

\section{Laxmilatha Pappurajam ${ }^{1}$, Shijin Ameri ${ }^{1}$, K A Labeeb ${ }^{1}$, A Kathirvel Pandian ${ }^{2}$, L Ranjith ${ }^{1}$ and A Gopalakrishnan $^{1}$}

${ }^{1}$ ICAR - Central Marine Fisheries Research Institute, P B No. 1603, Ernakulam North P O, Kochi 682018, Kerala, laxmilatha.p@icar.gov.in

${ }^{2}$ ICAR-National Bureau of Fish Genetic Resources, Kochi Unit, CMFRI Campus, P B No. 1603, Ernakulm North P O Kochi 682018, Kerala

The cone snails are hyper diverse group of marine gastropods and the shell morphology is the base of primary species identification. Among the Conidae, homoplasy and plasticity of morphological characters are common. Since speciation is positively correlated with peptide complexity in Conidae family, the present study was carried out to ascertain the genetic status of drastically varying phenotypes observed among the targeted Conus species of different environments and to detect cryptic species using mitochondrial and nuclear markers. Morphological parameters were recorded for the phenotypes of the species. Specific differences were observed for shell coloration. Three mitochondrial genes, COI (658bp), 16s rRNA (550bp), 12s rRNA (450bp) and Nuclear H3 gene (350bp) were amplified in five specimens each of varying phenotypes of targeted species. The analysis revealed that the phenotypic variations in C. inscriptus C.amadis and C.malacanus in different environments are not underpinned by the genome, The present study generated species-specific molecular signatures from varying phenotypes of conidae Since speciation is positively correlated with the complexity of conopeptides of the species, the findings provide confirmation of phenotypic variation and will be used for bioprospecting research on the species and also conservation and management of the species in Indian waters. 